\title{
Gas hydrates in coal seams and their impact on gas-dynamic processes in underground mining
}

\author{
Tatyana $\mathrm{Kim}^{1,{ }^{*}}$, Valery Dyrdin ${ }^{1}$, Vyacheslav Smirnov $^{1}$ and Valery Nesterov $^{2}$ \\ ${ }^{1}$ T.F. Gorbachev Kuzbass State Technical University, Department of physics, 650000 Kemerovo, 28 \\ Vesennaya st., Russian Federation \\ ${ }^{2}$ T.F. Gorbachev Kuzbass State Technical University, Department of mining machines and systems, \\ 650000 Kemerovo, 28 Vesennaya st., Russian Federation
}

\begin{abstract}
This paper deals with the development and analysis of the method of predicting gas-dynamic phenomena due to the gas hydrates in coal seams. With the help of the method of mathematical modelling the rate of dissociation of gas hydrates depending on the size of particles was determined as well as the correlation between the speed of moving the boundary line of the dissociation of gas hydrates in the pore space of coal and that of extending of the wave of crushing. The regularity of gas pressure distribution in a coal seam marginal zone after dissociation of gas hydrate depending on its saturation capacity and taking into account the changing coefficient of permeability of this zone was stated. It was also found that this kind of gas-dynamic processes in the presence of gas hydrates in the pore space of coals depends on critical value of permeability of a coal seam marginal zone.
\end{abstract}

\section{Introduction}

The main objective of this research is to determine the safe working conditions of Kuzbass coal miners namely to develop the method of predicting gas-dynamic phenomena (spontaneous gas emission and sudden coal and gas outburst) due to the gas hydrates, dissociation of which produces extra gas volume making major contribution to the gas balance of the bottom-hole zone of a coal seam thus increasing prediction accuracy. In coal seams the natural gas can be found in different states: free, retained and in the form of a solid coal gaseous solution of the natural gas like gas hydrates [1-4]. Phase transitions from a solid phase into a free one and vice versa are possible providing that thermodynamic parameters are bigger or smaller than the equilibrium ones. Gas hydrates are inclusion compounds where methane molecules are embedded into a water molecules frame. When the dissociation of gas hydrates occurs, extra gas volume is released influencing the formation of different kinds of gas-dynamic phenomena. Present methods of predicting the gas-dynamic phenomena for example, spectro-acoustic method of outburst prediction, do not take into account the release of extra gas volumes due to the dissociation of gas

\footnotetext{
*Corresponding author: tanyakim@list.ru
} 
hydrates as well as the increasing of the gas pressure which is an active force of the gasdynamic phenomena development.

\section{Materials and Methods}

From works [5-9] it follows that in coal seams under certain thermobaric conditions and humidity at some distance from the mine face there can be the areas containing a solid coal gaseous solution.

If there is a sharp stress drop caused by the mine face getting into a zone of increased rock pressure, or into a zone of a coal seam with low strength properties, in a thin coal seam in advance of the face under the action of the tensile stress there could appear the flaws in the pores in a plane orthogonal to a direction of the least squeezing that is parallel to the surface of the mine face. The extending of small flaws causes a drop of gas pressure to a state below the equilibrium. This provokes the beginning of two processes: gas desorption from the surface of macro pores and flaws, and also a dissociation of gas hydrates which lead to the release of extra gas volumes, the increase of the gas pressure in a marginal zone and the formation of gas-dynamic phenomena.

\section{Results and Discussion}

The possibility of methane gas hydrates formation in the coal under the low humidity has been tested at the Nikolaev Institute of Inorganic Chemistry (Russian Academy of Sciences) in the laboratory of clathrate compounds. It has been found that gas hydrates are formed in a coal matrix providing the thermobaric parameters determine the state of system above an equilibrium curve, and the rate of moisture is $1.5 \%$ or more. In this case the solid coal gaseous solution is formed in pore channels and evenly distributed in the form of crystals to the entire volume of coal providing the given parameters. So, it has been experimentally proved that the formation of solid natural gas solutions in coal pores can occur at moisture level characteristic for the coal seams dangerous because of the gas dynamic phenomena. To define the possible influence of solid coal gaseous solutions on extra gas emission from the solid block we set a task to determine the dissociation rate of gas hydrates depending on their sizes and allocation in a coal matrix. Drilling and maintenance of the deep oil and gas holes shows that the formation of gas hydrates in solid mass at their bottom leads to stopping of the process [10-14], as the speed of their decomposition is connected with the lowering the temperature of the environment. In this regard, we determine the gas hydrate dissociation rate in a coal seam, that is we determine how fast the decomposition into gas and water of a gas hydrate particle with the size corresponding to the size of pores in the structure of coal takes place. Let us take $T-$ current temperature of coal; $T_{0}=278 \mathrm{~K}$ - the temperature at which the decomposition of a gas hydrate takes place; $r_{2}$-radius of a gas hydrate particle.

According with the Fourier equation, the heat flow from coal in solid to a gas hydrate particle equals

$$
d Q_{1}=\chi \cdot \frac{\partial T}{\partial r} \cdot 4 \pi r^{2} d t
$$

where $\chi$ - thermal conductivity of coal;

The heat necessary for gas hydrate dissociation equals:

$$
d Q_{2}=\lambda \cdot d m,
$$


where $\lambda=533 \cdot 10^{3} \mathrm{~J} / \mathrm{kg} \quad-$ specific heat of gas hydrate decomposition, $d m=4 \rho_{1} \pi r_{2}^{2} d r_{2}$ - the mass of decomposing gas hydrate; $\rho_{1}=840 \mathrm{~kg} / \mathrm{m} 3-$ density of methane gas hydrate.

Equaling the expressions (1) and (2), we get

$$
\frac{d r_{2}}{d t}=\frac{\chi}{\lambda \rho_{1}} \cdot \frac{r_{1}^{2}}{r_{2}^{2}} \frac{\partial T}{\partial r_{1}}
$$

where $\frac{d r_{2}}{d t}=v$ - rate of gas hydrate particle dissociation in the block of coal mass.

It was experimentally stated that in the process of gas hydrate particles decomposition temperature $T$ recovers for the fractions of seconds thanks to the heat influx from the surrounding rock mass.

Gas hydrate particles in the coal mass have structure of Type-I which is close to the spherical structure.

Taking into consideration initial and marginal conditions for the chosen model, we have the temperature of the coal mass at the initial time equal: $T\left(r_{1}, 0\right)=T_{1}$, and the temperature of a gas hydrate particle at the beginning of decomposition also equal $T_{1}$, i.e. $\left.T\left(r_{2}, 0\right)\right|_{r_{2}=r_{1}}=T_{1}$. With the help of mathematical modelling we got the dependence of rate of gas hydrate particles dissociation from their sizes (fig.1.) by reference to the characteristics of methane gas hydrate and coal $[15,16]$.

The rate of a dissociation of gas hydrates nonlinearly depends on sizes of dissociating particles and if the diameter is 4 nanometers, it is equal to 28 nanometers/ms while with the increase of the size of gas hydrate particles the rate of a dissociation decreases. It is significant that the module of velocity of a hydrate dissociation in pore space of coal considerably surpasses the velocity of a crushing wave (A. S. Khristianovich). As the natural moisture content of coal seams, dangerous and endangered by gas-dynamic phenomena, is not more than $1.5-2.0 \%$, so gas hydrate particles are at a certain distance from each other, and their dissociation rate depends on their size only, i.e. the size of the pore channels, where they are formed.

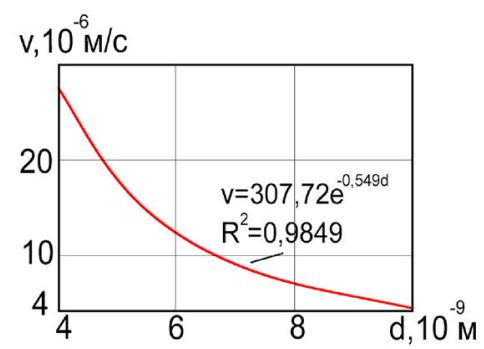

Fig. 1. Dependence of rate of gas hydrate particles dissociation from their sizes d-size of a gas hydrate particle

The dissociation of clathrate hydrates in the solid rock at the initial time occurs near the boundary of the inelastic and elastic deformations and eventually moves deep into the rock mass. The rate of moving the dissociation boundary line we find from the solution of the differential equation by A. M. Maximov: 


$$
\left.k_{1} \cdot\left(\frac{\partial p_{1}}{d x}\right)\right|_{x=\xi}-\left.k_{2} \cdot\left(\frac{\partial p_{2}}{\partial x}\right)\right|_{x=\xi}=\frac{W \mu p_{0}}{p_{p}} \cdot \frac{d \xi}{d t}
$$

And the current pressures $p_{1}, p_{2}$ in the areas of inelastic and elastic deformations (areas 1 and 2) we define from the equation of methane moving in a layer of onedimensional isothermal flow, where $p_{0}$ - atmospheric pressure, $\mathrm{Pa} ; \mu$-dynamic viscosity of the gas, $\mathrm{Pa} \cdot \mathrm{s} ; \varsigma$ - boundary line coordinate of hydrate decomposition, $\mathrm{m}$;

$W$ - the content of gas (hydrated state) depending on the porosity and moisture of the coal seam in a unit volume of the porous environment; $k_{1}, k_{2}$ - permeability coefficients in the areas 1 and 2, darcy; $p_{p}$ - hydrate decomposition pressure at seam temperature, $\mathrm{Pa}$.

The content of a gas (hydrate state) is the following:

$W=n(x)=n_{0}\left(1-e^{-b x}\right) \cdot V_{0}^{1}$, where $n(x)$ - function of particles quantity of the distance, $V_{0}^{1}-$ Volume of one particle of a gas hydrate, $n_{0} \cdot V_{0}^{1}=n_{0} \cdot \frac{4}{3} \cdot \pi \cdot r^{3}$; given that $r=\sqrt{\frac{S_{n o b}}{4 \pi}}$, and $W$ can be found from the formula: $W=\frac{n_{0} S}{3 V} \sqrt{\frac{S}{4 \pi}\left(1-e^{-b x}\right)}$, where $n_{0}$ concentration of gas hydrate particles.

The given task was solved for the seam "Vladimirovsky" of "Severnaya" mine and was reported by the authors in. Finding from (4) $v=\frac{d \xi}{d t}$ we determine the rate of movement of gas hydrate dissociation boundary for different decomposition pressures characteristic for coal seams with different thermodynamic parameters. The results of calculation are given in fig.2.

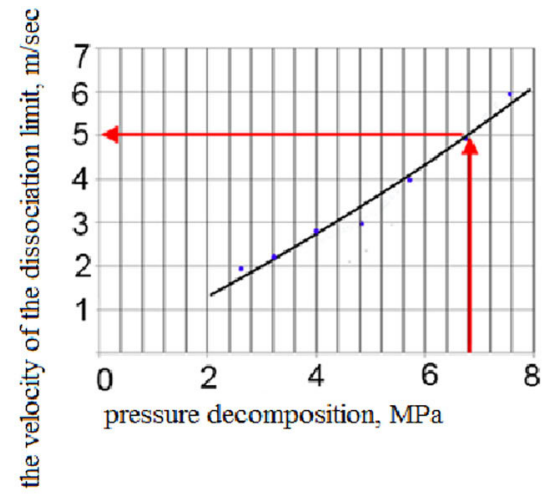

Fig. 2. Rate of movement of gas hydrate dissociation boundary for different decomposition pressures

Analyzing the found dependence we can see that the rate of movement of gas hydrate dissociation boundary for decomposition pressure equal $6.8 \cdot 10^{6} \mathrm{~Pa}$ is $5 \mathrm{~m} / \mathrm{s}$ and is comparable to the speed of extension of a crushing wave calculated according to the method of A. S. Hristianovich which is up to $5.95 \mathrm{~m} / \mathrm{s}$ for the given requirements. Thus, the crushing wave movement is accompanied by the decomposition of gas hydrates and the release of natural gas extra volume which accounts for the possible participation of gas hydrates in formation of the gas dynamic phenomena. 
The distribution of gas pressure in a gas hydrates decomposition ahead of a working face depending on its saturation capacity and taking into account the changing coefficient of permeability is also of great interest.

In solving this task, we suppose that at the maximum point of the zone of bearing pressure, we have the minimum permeability of rock mass. Out of the zone of mine entry influence the coefficient of permeability takes constant value $k_{\infty}$.

The other boundary conditions for the permeability coefficient are:

a) permeability at a development face $\left.k(x)\right|_{x=0}=k_{0}$, i.e. the beginning of the $\mathrm{x}$ axis coincides with a development face of a mine working and $x>0$ - on the depth of rock mass;

b) $\left.\quad k_{3}(x)\right|_{x=\xi}=\left.k_{4}(x)\right|_{x=\xi}=k$, where $x=\xi$ - distance from the mine face to the beginning of the zone, where the gas hydrates dissociation takes place, i.e. it is assumed that the dissociation of clathrate hydrates begins at the boundary of the elastic $\left(x_{3}\right)$ and inelastic $\left(x_{4}\right)$ deformations.

Methane movement in a coal seam is presented by the Bussinesk differential equation for the one-dimensional isothermal flow gained by L. S. Lejbenzon:

$$
\frac{\partial p}{\partial t}=\frac{k}{2 m \mu_{g}} \cdot \frac{\partial^{2} p^{2}}{\partial x^{2}},
$$

$m$ - porosity coefficient of environment; $\mathrm{p}$ - pressure of gas; $\mu_{g}$-dynamic viscosity of gas; $k$ - coefficient of permeability. First of all, we define the number of moles $v$ of the gas present in hydrated state in a coal seam:

$$
v=\frac{V_{\text {coal }}(1-\varepsilon) \rho_{\text {hydrate }}}{\mu_{\text {water }} n} \beta \text {, where } \beta=\frac{W}{1-\varepsilon} \frac{\rho_{w}}{\rho_{h}}-\text { saturation capacity of hydrate; }
$$

Water density $-\rho_{w}=1000 \mathrm{~kg} / \mathrm{m}^{3} ; \varepsilon=0.126-$ natural mass of gas in a mass unit of gas hydrate; density of hydrate of rock gas $\rho_{h}=883 \mathrm{~kg} / \mathrm{m}^{3} ; W$ - natural moisture of coal; $\mu_{\text {water }}$ - molar mass of water; $n$ - quantity of molecules of water on one molecule of gas in each mesh of gas hydrate of Type-1.

As an example, we have calculated the distribution of gas pressure after the beginning of the dissociation process for the "Vladimirovskiy" seam of "Severnaya" mine. The results are given in Fig. 3.

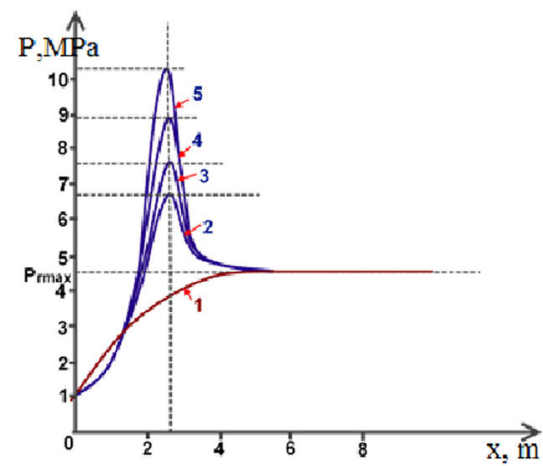

Fig. 3. The distribution of gas pressure after the beginning of the gas hydrates dissociation process and changing permeability of a boundary zone of a coal seam: 1 - the initial distribution of the gas pressure; 2 - hydrate saturation at $1.9 \% ; 3$ - hydrate saturation at $2.6 \%$; 4 - hydrate saturation at $3.9 \%$; 5 - hydrate saturation at $5.2 \% ; \mathrm{P}_{\max }-$ gas pressure out of the zone of mine entry influence 
The extra volume of the free gas depends on extent of the area containing gas hydrates, i.e. on its hydrate saturation. Thus, when the inherent humidity of a seam is from 1.5 to $2.5 \%$, additional gas pressure in the zone of gas hydrates decomposition increases by $2-2.5$ times directly affecting the increase of gas pressure ahead of a working face and can cause spontaneous gas emission and sudden coal and gas outburst.

To determine the type of gas-dynamic phenomena (spontaneous gas emission or gas hazard), we calculate the hazard ratio of emission gas in the reservoir $R^{n}$ by the method of prof. V.I. Murashev, taking into account the pressure rise due to the dissociation of gas hydrates of methane, and by the transition point of the curve through zero we define critical $\mathrm{k}_{k p}$ for the given seam.

To define the permeability coefficient in practice, we assume that in the marginal zone of a coal seam ahead of a working face it varies according to the law:

$$
k(x)=k_{0} e^{-b \cdot \sigma(x)},
$$

where $k_{0}$ - coefficient of permeability of coal in the initial state, $\mathrm{m}^{2}$ (determined in the laboratory conditions by the method of V.V. Hodot); b - a constant which depends on the compression properties of the coal seam; $\sigma(x)$ - tension at the point considered.

It is necessary to know the coefficient of permeability of rock mass at the boundary line of inelastic and elastic deformations where the clathrate hydrates dissociation begins. The distance to the boundary line is determined from the inflection point of the experimental culm yield curve in drilling wells and the stresses at this point are found in accordance with their linear approximation in the zone of maximum stress state. For this purpose we determine the stresses at the bottom and at the peak of the bearing pressure according with the well-known formulas of VNIMI.

For the values of the permeability coefficient $k$ at the interface between elastic and inelastic deformations exceeding the critical value for the given coal seam, gas content production occurs (since there is an intensive gas filtering to the mine face) and for $k<k_{k r}$ outburst situation is formed.

As an example, for the seam "Vladimirovskiy" of the mine "Severnaya" it was calculated that the practical value of the permeability coefficient at $l=3.5 \mathrm{~m}$ is $310^{-15} \mathrm{~m}^{2}$, and the critical value for these conditions is $10 / 10^{-15} \mathrm{~m}^{2}$, i.e.

$k_{p r}\left(3.5=3 \cdot 10^{-15} \mathrm{~m}^{2}<k_{\kappa p}=10 \cdot 10^{-15} \mathrm{~m}^{2}\right.$, so this section is prone to spontaneous outbursts of coal and gas (Figure 4).

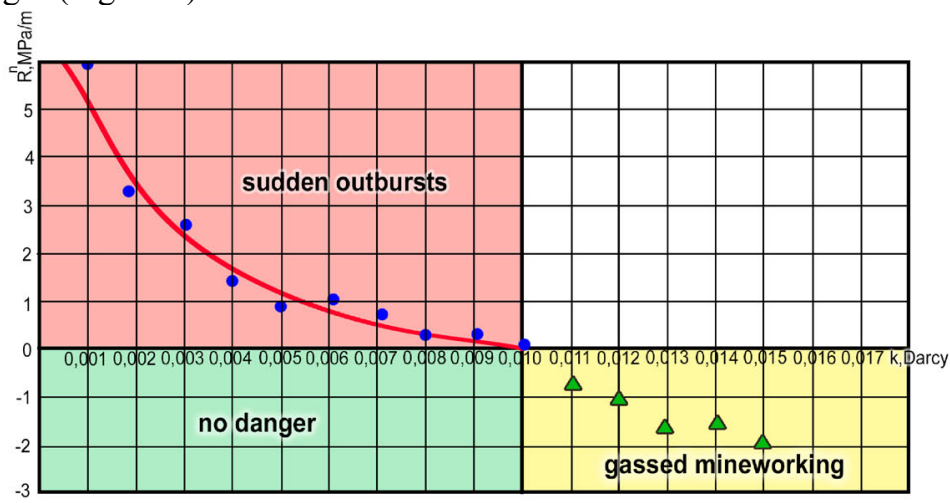

Fig. 4. Dependence of the gas dynamic phenomena types on the rock mass permeability at the interface between elastic and inelastic deformation 


\section{Conclusions}

This technique allows us to identify areas in coal seams prone to gas-dynamic phenomena taking into account the possibility of methane gas hydrates decomposition and to improve the reliability of the current forecast of dangerous coal and gas outbursts by taking into account the dissociation of gas hydrates, which are the major contributors in the gas balance in the process of gas-dynamic phenomena formation.

\section{References}

1. Yu.A. Dyadin, E.G. Larionov, A.Y. Manakov, E.Y. Aladko, Mendeleev Communications, 209 (1999)

2. Melnikov V.P., Nesterov A.N., Podenko L.S., Reshetnikov A.M., Shalamov V.V., Chemical Engineering Science, 71, 573 (2012)

3. Yao SuPing, Chinese Science Bulletin, 25, 2706 (2011)

4. Sloan, E. D. Clathrate Hydrates of Natural Gases - 3rd ed., (CRC Press, 2008)

5. V.V. Dyrdin, V.G. Smirnov, S.A. Shepeleva, Journal of Mining Sci, 49, 908 (2013)

6. V.G. Smirnov, A.Y. Manakov, E.A. Ukraintseva, V.V. Dyrdin, I.S. Terekhova, A.G. Ogienko, Fuel, 166, 188 (2016)

7. Clarkson, C. R., R. M. Bustin, Fuel, 78, 1333 (1999)

8. Christophe Dicharry, Pascal Gayet, Gerard Marion, Alain Graciaa and Anatoliy N. Nesterov, J. Phys. Chem., 109, 17205 (2005)

9. Lijun Yan, Guangjin Chen, Weixin Pang, J. Phys. Chem., 109, 6025 (2005)

10. Liu, J, Jiang X, Huang X, et al. Fuel, 89, 3884 (2010)

11. Skoczylas N., Arch Min Sci, 57, 861 (2012)

12. Sobczyk J., Fuel, 90, 1018 (2011)

13. Yi J, Akkutlu IY, Karacan CÖ, Clarkson CR., Int J Coal Geol, 77, 137 (2009)

14. Jianming, Yu Bufan, Wang Youan, al. The technology hand book of coal and gas outburst control (China University of Mining and Technology Press, 2006)

15. Lama, R. D., Australia, Report GSM21 (1981)

16. State Administration of Quality and Technical Supervision, State Administration of Coal Mine Safety. Prevention of Coal and Gas Outburst Provision (Beijing: ChinaCoal Industry Publishing House, 2009) 